\title{
Reliable navigation planning implementation on a two-wheeled mobile robot
}

\author{
Pierre-André Crépon \\ ENSTA ParisTech, \\ Palaiseau, France \\ crepon.pierre.andre@gmail.com
}

\author{
Adina M. Panchea \\ LIX, École Polytechnique, \\ Palaiseau, France \\ panchea@lix.polytechnique.fr
}

\author{
Alexandre Chapoutot \\ U2IS, ENSTA ParisTech, \\ Palaiseau, France \\ alexandre.chapoutot@ensta-paristech.fr
}

\begin{abstract}
Autonomous mobile robots must be equipped with appropriate planification and control navigation systems in order to obtain robust behaviours. This study aims at dealing with this kind of problems when implementing on a two wheeled mobile robot. The planning navigation system uses our previously proposed reliable and safe navigation planning algorithm based on the incremental sampling-based planning algorithm, e.g., the widely-used Rapidly-exploring Random Tree (RRT), while the control navigation level consists in a go to goal controller strategy. Through experiments, we demonstrate the usefulness of robust navigation planner in an autonomous navigation schemes, where uncertain localization has to be taken into account.
\end{abstract}

\section{INTRODUCTION}

One of the objectives in the field of the autonomous navigation of mobile robots is the development of mobile platforms that robustly operate in complex or populated environments while offering various services without human interactions. A challenge for the autonomous navigation's planner level is related to the guarantee of the system's safety. To this end, in this study, we use a previously proposed reliable motion planner algorithm [3] based on Rapidly-exploring Random Trees (RRT) principles [1], which covers the whole configuration space and easily integrates complex robot models, and solved in an interval analysis [2] framework. More precisely, we consider while planning, the uncertainties from a visual localization provided by camera, bounded with know bounds. For navigation control algorithms an interesting survey is proposed in [4], while in this study a go to goal strategy is used. Briefly, the main contribution of this paper is the implementation on a real mobile robot application of an navigation planner under DynIBEX ${ }^{1}$, while the visual localisation information is bounded with known bounds.

\section{Problem Statement}

Context Given a mission, which consists in starting from an initial and a goal configurations provided by a visual sensor (camera) to the motion planning level, waypoints are computed and then sent to the controller level so that a mobile robot can reach the goal point while avoiding static obstacles.

Problem Given unique initial and goal positions, find collision-free paths and drive a mobile robot from the initial to the goal position.

\footnotetext{
${ }^{1}$ http://perso.ensta-paristech.fr/ chapoutot/dynibex/
}

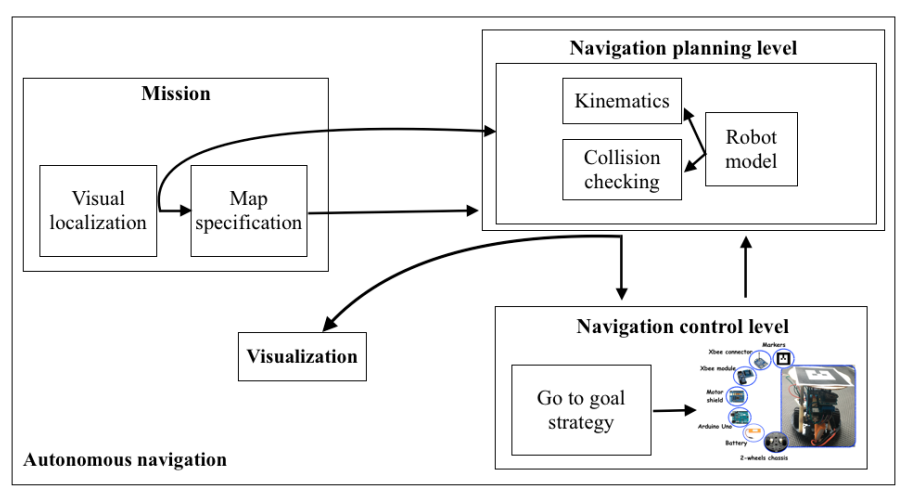

Figure 1. Autonomous navigation architecture

Experimental architecture: The mobile robot has to be driven in a two-dimensional static configuration space of size $1.2 \mathrm{~m} \times 1.8 \mathrm{~m} \times 2 \pi \mathrm{rad}$, from an initial state to a desired one while avoiding polygons shaped obstacles. A brief description of the experimental navigation architecture is given in Fig. 1 and it is as follows: First, the static configuration space, with free and non-admissible region subsets, is provided. Then, a $\mathrm{QR}$ code tracking package along with visual sensor ( $a$ camera), is used to specify the initial and final configurations. Then, for the given configuration space the planner will generate waypoints for a collision-free path and send it to the Arduino board via radio communication server based Xbee modules which will make the mobile robot reaching the final region subset.

Solution First we create a two-wheel mobile robot configuration which is localized by a visual sensor, receives the commands information via radio transmission from the navigation planning level sends sequence of control policy or waypoints given by the robust navigation planner, namely BoxRRT. Hence the mobile robot can reach to the desired configuration following the architecture illustrates on Fig. 1.

\section{NAVIGATION PLANNING LEVEL}

First the given initial configuration $\left[\mathbf{s}_{\text {init }}\right]$ is added to the exploration tree $G$ (Line 1$)$. Then, a state $\left[\mathbf{s}_{\text {rand }}\right] \subset \mathbb{S}_{\text {free }}$ is randomly chosen by the procedure random-box-GoalBias (Line 4). The nearest-neighbor procedure from Line 5 returns the closest vertex $\left[\mathbf{s}_{\text {near }}\right]$ to $\left[\mathbf{s}_{\text {rand }}\right]$ in the tree $G$, according 
the Hausdorff distance between two intervals metric $d$. The select input procedure provides a control input $\mathbf{u}=[v \omega] \in[\mathbf{u}]$, with $v$ the linear and $\omega$ the angular speed. The control input is exhibited by using a go-to-goal principle by orienting the mobile robot towards the target and going straight ahead until it's reaching the goal. Then, in the prediction procedure, the differential system which can the evolution of the mobile robot system: $\dot{s}=f(s(t), u(t))$ is integrated over a fix time interval $\Delta t$ with the initial condition $\left[\mathbf{s}_{\text {near }}\right]$ and a constant control input $\mathbf{u}$ (given at Line 6) and will result in a new state $\left[\mathbf{s}_{\text {new }}\right]$ (Line 7). If it can be proved that all state values along the trajectory between $\left[\mathbf{s}_{\text {near }}\right]$ and $\left[\mathbf{s}_{\text {new }}\right]$ lie in $\mathbb{S}_{\text {free }}$ being a collision free path, then the path between $\left[\mathbf{s}_{\text {near }}\right]$ and $\left[\mathbf{s}_{\text {new }}\right]$ is considered reliable and $\left[\mathbf{s}_{\text {new }}\right]$ is added to $G$ as a new vertex and connected to its parent $\left[\mathbf{s}_{\text {near }}\right]$ though the G.add-guaranteed-vertex procedure. Otherwise, $\left[\mathbf{s}_{\text {new }}\right]$ is not added to $G$. Lines 4 to 11 are repeated until a chosen number of iterations $\mathrm{K}$ is reached or until a path is found meaning $\left[\mathbf{s}_{\text {new }}\right]=\left[\mathbf{s}_{\text {goal }}\right]$, or most likely when $\left[\mathbf{s}_{\text {new }}\right] \subset\left[\mathbf{s}_{\text {goal }}\right]$. Note that we have $\left[\mathbf{s}_{\text {init }}\right]=\operatorname{Hull}\left(\mathbb{S}_{\text {init }}\right),\left[\mathbf{s}_{\text {obs }}\right]=\operatorname{Hull}\left(\mathbb{S}_{\text {obs }}\right)$ and $\left[\mathbf{s}_{\text {goal }}\right]=\operatorname{Int}\left(\mathbb{S}_{\text {goal }}\right)$ to ensure the soundness of the proposed algorithm.

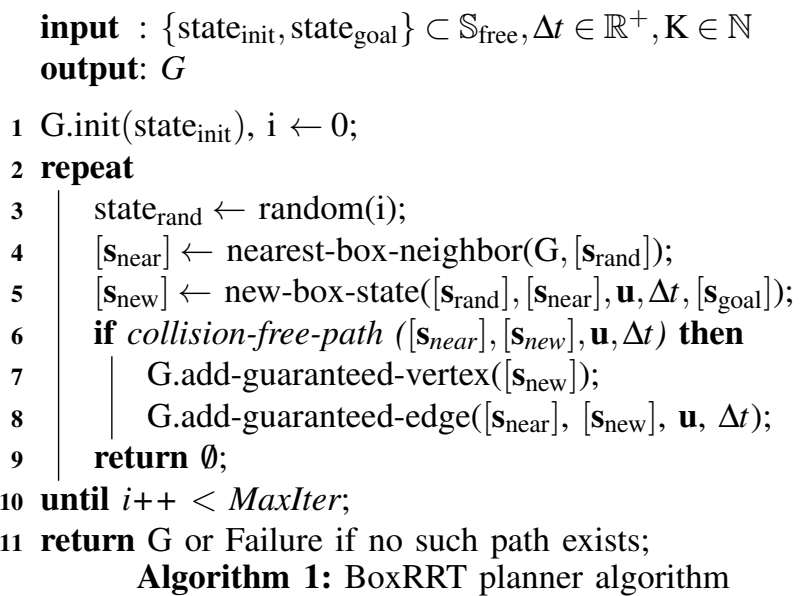

\section{EXPERIMENTAL RESULTS}

All simulations and experimental tests were performed on an Intel Core $\mathrm{m} 7-6 \mathrm{Y} 75 \mathrm{CPU}$ at $1.20 \mathrm{GHz} \times 4$. The used software for the BoxRRT planner consists in DynIBEX, while the middleware used for the experimentations consists in Robot Operating System (ROS).

The differential system which describes the evolution of the mobile robot is represented by the kinematic model of an unicycle robot type: $\dot{x}=v \cos \theta, \quad \dot{y}=v \sin \theta, \quad \dot{\theta}=\omega$, with $(x y)$ the position and $\theta$ the orientation w.r.t. a frame attached to the environment. The control input $u=[v \omega]$ is represented by the linear $v \in[-0.4 ; 0.4] \mathrm{m} / \mathrm{s}$ and the angular $\delta \in[-10 ; 10]$ $\mathrm{rad} / \mathrm{s}$ velocities respectively. The latter values were determined experimentally.The reported CPU values are the mean of the CPU obtained after 100 iterations. Although, the obtained CPU performances for all three investigated configurations



Figure 2. Planning visualization

remains less than 70s. For all trials the navigation planners found waypoints for free-collision paths. The purpose of this study is to introduce the BoxRRT navigation algorithm for real application and to presents the advantages of this planner usage, when using localisation information provided by visual means and which depends on QR code can be perturbed due to some external perturbations. In the latter hypothesis and if one utilises the navigation planners provided by the OMPL [5] the mobile robot needs to be physically placed at the precise desired location. While for the BoxRRT planner an imprecise initial position can be fixed and a tube is provided without no user intervention. Moreover, as long as the robot stays inside the provided tube of trajectories we are certain that the robot achieves the task with no collision.

\section{CONCLUSIONS AND FUTURE WORKS}

In this study, our previously proposed BoxRRT navigation planner is implemented on a real mobile robot application for the first time. In future work we plan to provide the robust and reliable motion planner based on an online library.

\section{ACKNOWLEDGMENT}

This work was supported by DGA MRIS.

\section{REFERENCES}

[1] S. M. LaValle. Rapidly-exploring random trees: a new tool for path planning. Technical report, Iowa State University, 1998.

[2] R. E. Moore. Interval Analysis. Prentice-Hall, 1966.

[3] A. M. Panchea, A. Chapoutot and D. Filliat. Extended Reliable Robust Motion Planners. In $C D C, 2017$, to appear.

[4] B. Paden, M. Cap, S. Z. Yong, D. Yershov, E. Frazzoli A Survey of Motion Planning and Control Techniques for Self-Driving Urban Vehicles. IEEE Transactions on intelligent vehicles, 1(1):33-55, 2016.

[5] I.A. Şucan, M. Moll and L.E. Kavraki. The Open Motion Planning Library. IEEE Robotics \& Automation Magazine, 18(4):72-82, 2012. 